\title{
THE GROMOV NORM AND FOLIATIONS
}

\author{
DANNY CALEGARI
}

\begin{abstract}
We define a norm on the homology of a foliated manifold, which refines and majorizes the usual Gromov norm on homology. This norm depends in an upper semi-continuous way on the underlying foliation, in the geometric topology. We show that this norm is non-trivial - i.e. it distinguishes certain taut foliations of a given hyperbolic 3-manifold.

Using a homotopy-theoretic refinement, we show that a taut foliation whose leaf space branches in at most one direction cannot be the geometric limit of a sequence of isotopies of a fixed taut foliation whose leaf space branches in both directions. Our technology also lets us produce examples of taut foliations which cannot be made transverse to certain geodesic triangulations of hyperbolic 3-manifolds, even after passing to a finite cover.

Finally, our norm can be extended to actions of fundamental groups of manifolds on order trees, where it has similar upper semi-continuity properties.
\end{abstract}

\section{InTRODUCTION}

The study of group actions on trees and tree-like objects has for a long time been an important tool in 3-manifold topology. J. Stallings pioneered this approach with a topological proof of Grushko's theorem [23], and more generally it is by now a standard observation that a decomposition of a 3-manifold along an incompressible surface is "dual" to some action of $\pi_{1}(M)$ on a tree. M. Culler and P. Shalen [6] used the action of $\pi_{1}(M)$ for $M$ a hyperbolic manifold on the Bruhat-Tits tree of $S L(2, F)$, where $F$ is the function field of a curve in the $S L(2, \mathbb{C})$ representation variety of $\pi_{1}$, to obtain striking topological results about $M$. More generally, a sequence of hyperbolic (or merely negatively curved) structures on a fixed manifold which are not precompact in the Gromov-Hausdorff topology may be rescaled and filtered to give in the limit an action of $\pi_{1}(M)$ on an $\mathbb{R}$-tree (see e.g. F. Paulin [22]). In a 2-dimensional setting, this idea is implicit in Thurston's compactification of Teichmüller space by projective measured laminations [24.

However, the tree-like structures on which $\pi_{1}(M)$ acts in all these cases admit some kind of invariant measure structure. For a taut foliation or an essential lamination, the existence of such a transverse structure is rare, and leads to strong topological conditions on the underlying manifold. Consequently many "naturally occurring" actions of fundamental groups of 3-manifolds on non-Hausdorff simply connected 1-manifolds and order trees admit no invariant measure. Nevertheless, one would like to quantify the amount of branching of such trees in some natural 
way. In this paper, we introduce a norm on the homology of a foliated manifold, which is a refinement of the usual Gromov norm on homology, where one restricts the admissible chains representing a homology class to those which are transverse - that is, each singular map $\sigma: \Delta^{i} \rightarrow M$ in the support of an admissible chain must induce a standard foliation on $\Delta^{i}$, one which is topologically conjugate to an affine foliation. For a hyperbolic manifold $M^{n}$, the Gromov norm of $[M]$ is proportional to the volume of $M$, and for $n \geq 3$, a chain whose norm is close to the infimum actually "detects" the geometry of $\widetilde{M}=\mathbb{H}^{n}$ (this is just a restatement of Mostow's rigidity theorem). For a foliated manifold, the tension between the geometry of the ambient manifold and the local affine structure determined by the foliation can be used to show that the foliated norm differs from the usual norm in certain cases, which reflect the topology and the geometry of the foliation.

In particular, we have the following theorems:

Theorem 2.2.10. Let $\mathcal{F}$ be a foliation of $M^{n}$ whose universal cover is topologically conjugate to the standard foliation of $\mathbb{R}^{n}$ by horizontal $\mathbb{R}^{n-1}$ 's. Then

$$
\|[M]\|_{G}=\|([M], \mathcal{F})\|_{F G}
$$

Theorem 2.5.9. Suppose that $\mathcal{F}$ is a taut foliation with one-sided branching. Then there is an equality of norms

$$
\|[M]\|_{G}=\|([M], \mathcal{F})\|_{F G}
$$

Theorem 2.4.5. Suppose $M^{n}$ is hyperbolic and $\mathcal{F}$ is asymptotically separated. Then

$$
\|[M]\|_{G}<\|([M], \mathcal{F})\|_{F G}
$$

Here we say that a foliation $\mathcal{F}$ of a hyperbolic manifold is asymptotically separated if for some leaf $\lambda$ of $\widetilde{\mathcal{F}}$, there are a pair of open hemispheres $H^{+}, H^{-} \subset \mathbb{H}^{n}$ in the complement of $\lambda$ which are separated by $\lambda$. We point out that a standard conjecture would imply that a taut foliation of a hyperbolic 3-manifold is asymptotically separated iff $\widetilde{\mathcal{F}}$ has two-sided branching.

It should be mentioned that a norm for foliations with transverse measures was defined by Connes (unpublished) and developed in [15] and [16]. This norm uses generalized simplices which are simplicial in the tangential direction and measuretheoretical in the transverse direction. It has the usual proportionality properties for foliations whose leaves are all locally isometric to a space of constant curvature. The "fundamental cycle" on which this norm is evaluated is really attached to the 
measured foliation, and not to the underlying manifold. By contrast, our definition is closer in spirit to norms for stratified or decorated spaces.

With our definition, the norm on a homology class is upper semi-continuous as a function of the underlying foliation, in the geometric topology. Since the norm is defined topologically, this gives obstructions for the existence of a family of isotopies of a fixed topological foliation to converge geometrically to some other foliation. The leaf space of the universal cover of a taut foliation is a (typically non-Hausdorff) simply-connected 1-manifold. The non-Hausdorffness comes from branching of the leaf space. This branching can occur in both directions, in only a single direction, or not at all. The taut foliation is said in these three cases to have branching in both directions, to have branching in only one direction, or to be $\mathbb{R}$-covered. We show

Corollary 3.1.5. Let $\mathcal{F}$ with branching on at most one side and $\mathcal{G}$ with two-sided branching, be taut foliations of $M^{3}$. Then there is no sequence of isotopies $\mathcal{G}_{i}$ of $\mathcal{G}$ which converges geometrically to $\mathcal{F}$.

Using similar techniques, we show

Theorem 3.3.2. Let $M$ be a hyperbolic 3-manifold, and $\mathcal{F}$ any taut foliation with 2 -sided branching. Then there is a geodesic triangulation $\tau$ of $M$ which cannot be made transverse to $\mathcal{F}$. Furthermore, $\tau$ cannot be made transverse to $\mathcal{F}$ in any finite cover (i.e. $\tau$ is not virtually fine).

Problem 3.16 in Kirby's problem book 19] asks for a reasonable real-valued function on the set of 3-manifolds which measures the complexity of $\pi_{1}(M)$ and behaves appropriately under finite covers and positive degree maps. One may translate this problem into the foliated context, where one considers pairs $(M, \mathcal{F})$, finite covers and transverse positive degree maps (a map $f:(M, \mathcal{F}) \rightarrow(N, \mathcal{G})$ between foliated manifolds is transverse if every transversal to $\mathcal{F}$ is mapped to a transversal to $\mathcal{G}$ ), in which context our norm seems like a "reasonable" solution.

I would like to thank I. Agol, A. Casson and W. Thurston with whom I had some interesting discussions about this material. In particular, I. Agol's work [1] on volumes of hyperbolic 3-manifolds with boundary was particularly inspiring. Furthermore, I received partial support from a Sloan Dissertation Fellowship and from the Clay Mathematical Institute while carrying out work on this paper.

\section{Foliated NORMS}

2.1. Foliations. We give the basic definitions of various kinds of foliations of $3-$ manifolds. More details are to be found in [9]. 
Definition 2.1.1. Let $M$ be the subspace of $\mathbb{R}^{3}$ for which $z \geq 0$, minus the origin. $M$ has a foliation $\widetilde{\mathcal{F}}$ by leaves of the form $z=$ const. which are all planes, except for the leaf $z=0$ which is a punctured plane. This foliation is preserved by the dilation $(x, y, z) \rightarrow(2 x, 2 y, 2 z)$ and so descends to a foliation of the solid torus. This is called the Reeb foliation of the solid torus.

Definition 2.1.2. A codimension 1 foliation of a 3-manifold is Reebless if it has no solid torus subsets foliated with a Reeb foliation.

For Reebless foliations, every leaf is incompressible and the ambient manifold is irreducible or covered by $S^{2} \times S^{1}$ foliated by horizontal spheres. Moreover, every loop transverse to a Reebless foliation is homotopically essential. It follows that a Reebless foliation of a manifold pulls back in a finite covering to a co-orientable foliation, one for which there is a choice of orientation on transversals which is invariant under leaf-preserving isotopy. Equivalently, there is a $\pi_{1}(M)$-invariant orientation on the leaf space in the universal cover.

Definition 2.1.3. A codimension 1 foliation of a 3-manifold is taut if there is a circle in the manifold transverse to the foliation which intersects every leaf.

Every taut foliation is Reebless. The induced foliation $\widetilde{\mathcal{F}}$ of the universal cover $\widetilde{M}$ of a Reebless foliation is (topologically) a foliation of $\mathbb{R}^{3}$ by planes, which is the product of a foliation of $\mathbb{R}^{2}$ by lines with $\mathbb{R}$. Consequently, every plane is properly embedded and separates $\mathbb{R}^{3}$ into two topological half-spaces.
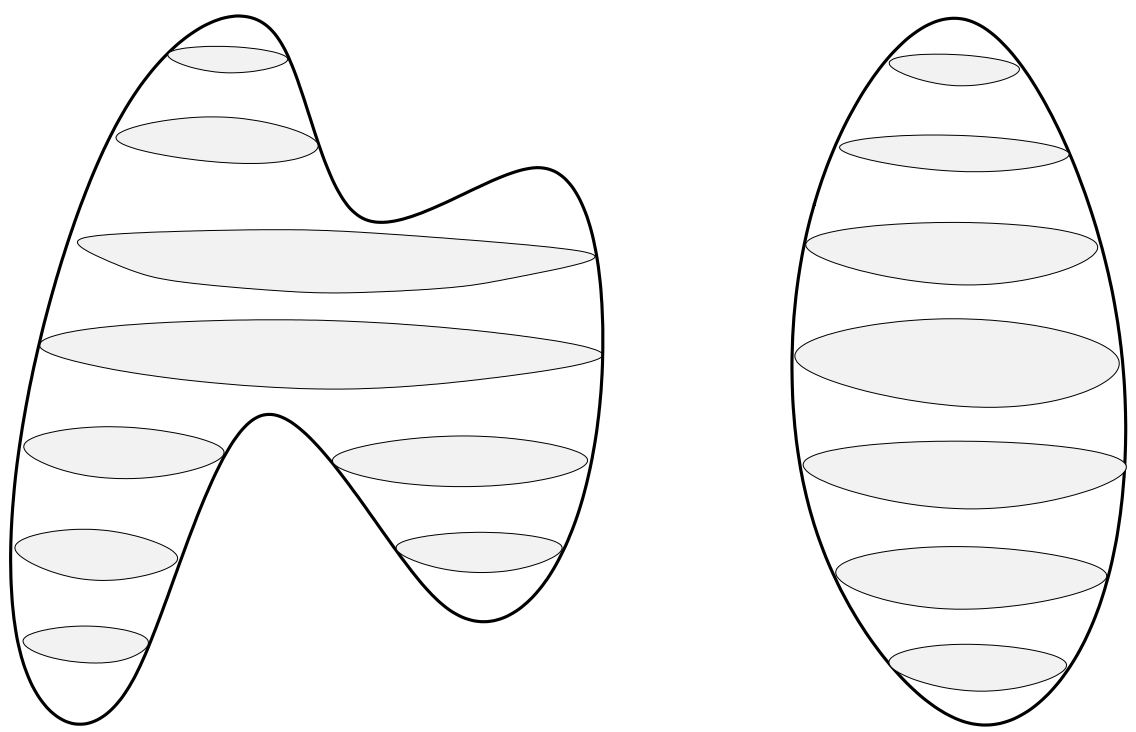

Figure 1. The foliation in the universal cover of two foliations: one $\mathbb{R}$-covered, the other not. 
The leaf space $L$ of the universal cover of a taut foliation is a (possibly nonHausdorff) simply connected 1-manifold. The orientation on this leaf space induces a partial order on the leaves: $\lambda>\mu$ iff there is a positively oriented transversal in $\widetilde{M}$ from $\mu$ to $\lambda$. The absence of loops in the leaf space make this partial order welldefined. For readers unfamiliar with the topology of non-Hausdorff 1-manifolds, consult [12]. The "non-Hausdorffness" arises from branching of the leaf-space: an embedded half-open arc in the leaf space might have a countably infinite collection of limiting endpoints. Moreover, this branching might take place at a dense set of points.

Definition 2.1.4. A taut foliation is $\mathbb{R}$-covered if the pulled back foliation of the universal cover is topologically conjugate to the standard foliation of $\mathbb{R}^{3}$ by horizontal planes.

For $M$ not finitely covered by $S^{2} \times S^{1}$, the leaf space of $\widetilde{\mathcal{F}}$ is Hausdorff exactly when $\mathcal{F}$ is $\mathbb{R}$-covered, for taut $\mathcal{F}$.

\subsection{Gromov norms.}

Definition 2.2.1. A singular $n$ chain in $M$ is a finite $\mathbb{R}$-linear combination of singular $n$-simplices, where a singular $n$-simplex is a map $\sigma: \Delta^{n} \rightarrow M$ from the standard affine $n$-simplex into $M$. The support of a chain, denoted $\operatorname{supp}(C)$ is the set of singular $n$-simplices with non-zero coefficients in $C$.

Notice that our convention is to assume that the coefficients in our chains are allowed to be in $\mathbb{R}$. We assume this without comment in the sequel.

The classical Gromov norm is defined in [14] as follows:

Definition 2.2.2. For $M$ an orientable $n$-manifold, let $[M]$ denote the fundamental class of $M$ in $H_{n}(M ; \mathbb{R})$. The Gromov norm of $M$ is the infimum of the $L_{1}$ norm on the singular cycles representing $[M]$. That is,

$$
\|[M]\|_{G}=\inf _{\left[\sum_{i} r_{i} \sigma_{i}\right]=[M]} \sum_{i}\left|r_{i}\right|
$$

Definition 2.2.3. For $M$ an orientable $n$-manifold and $\mathcal{F}$ a codimension $m$ foliation, call a singular cycle $\sum_{i} r_{i} \sigma_{i}$ transverse if the foliation on the $n$-simplex $\Delta^{n}$ induced from each singular map $\sigma_{i}: \Delta^{n} \rightarrow M$ by pulling back $\mathcal{F}$ is topologically conjugate to some affine foliation of $\Delta^{n}$ : that is, the foliation by preimages of points obtained from some affine map $\Delta^{n} \rightarrow \mathbb{R}^{n-m}$. The foliated Gromov norm of $[M]$ with respect to $\mathcal{F}$ is defined to be

$$
\|([M], \mathcal{F})\|_{F G}=\inf _{\left[\sum_{i} r_{i} \sigma_{i}\right]=[M] ; \sigma_{i}} \sum_{\text {transverse }} \sum_{i}\left|r_{i}\right|
$$

Remark 2.2.4. Notice that any map from the vertices of a simplex to $\mathbb{R}$ can be extended to an affine map of the simplex to $\mathbb{R}$. 

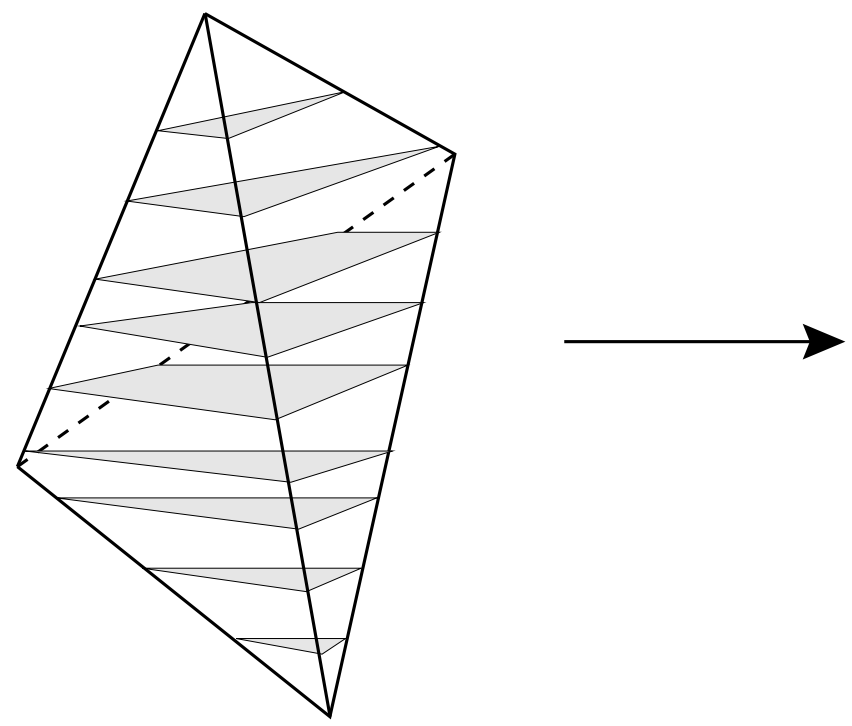

Figure 2. A map from the vertices of a simplex to $\mathbb{R}$ can be extended to an affine map, which pulls back to an affine foliation of $\Delta^{n}$.

Theorem 2.2.5. Let $M$ be a 3-manifold and let $\mathcal{F}$ be a taut foliation of $M$.

1. $\|([M], \mathcal{F})\|_{F G}<\infty$.

2. $\|[M]\|_{G} \leq\|([M], \mathcal{F})\|_{F G}$.

3. There is a $K(M)<\infty$ such that for any taut foliation $\mathcal{G}$ of $M,\|([M], \mathcal{G})\|_{F G}<$ $K(M)$. If $K(M)$ is the infimum of such, define $\|[M]\|_{F G}=K(M)$.

Proof: $\quad$ Fact 2. is obvious from the definition of the norms. Fact 1. follows from the existence of a triangulation of $M$ in which the foliation $\mathcal{F}$ can be put into normal form. Fact 3. follows from the stronger theorem of D. Gabai that on any 3 -manifold there is a triangulation with respect to which every taut foliation of $M$ can be put in normal form. ([1])

Remark 2.2.6. Of course, one can define any number of norms on homology by restricting the class of singular maps which are deemed admissible. The particular restriction of transversality seems suitable for studying foliations, since it behaves nicely with respect to many of the usual constructions of foliations, e.g. branched covers.

Lemma 2.2.7. Let $f: N^{n} \rightarrow M^{n}$ be a degree $d$ branched cover where the branch locus $\gamma$ is transverse to a foliation $\mathcal{F}$ of $M^{n}$. Let $\mathcal{G}$ be the foliation obtained from 
$\mathcal{F}$ by pullback. Then

$$
\|([N], \mathcal{G})\|_{F G} \geq d\|([M], \mathcal{F})\|_{F G}
$$

Proof: Let $C=\sum_{i} r_{i} \sigma_{i}$ be a transverse chain representing $[N]$. Then $f_{*} C=$ $\sum_{i} r_{i}\left(f \circ \sigma_{i}\right)$ is a transverse chain representing $d[M]$.

It is a well-known fact that the simplex is distinguished amongst all affine polyhedra by the fact that any total ordering of its vertices is induced from an affine map of the entire simplex to $\mathbb{R}$. In the context of foliations, this fact has the following generalization:

Lemma 2.2.8. Let $\mathcal{F}$ be a foliation of $M^{n}$ and let $C=\sum_{i} r_{i} \sigma_{i}$ be a cycle representing $[M]$. Suppose that the leaf space of $\widetilde{\mathcal{F}}$ is an acyclic 1-manifold and that the leaves of $\widetilde{\mathcal{F}}$ are all $\mathbb{R}^{n-1}$. Suppose further for each $i$ that $\sigma_{i}$ lifts to $\widetilde{\sigma}_{i}: \Delta^{n} \rightarrow \widetilde{M}$ such that the images of the vertices of $\Delta^{n}$ inherit a total order from the natural partial order on $L$. Then $C$ can be "straightened" to $C^{s}=\sum_{i} r_{i} \sigma_{i}^{s}$ where $\widetilde{\sigma}_{i}$ and $\widetilde{\sigma_{i}^{s}}$ have the same endpoints in $\widetilde{M}$, and $C^{s}$ is a transverse cycle.

Proof: $\quad$ Let $S_{n}=\operatorname{supp}(C)$ and inductively define $S_{i}=\operatorname{supp}\left(\partial S_{i-1}\right)$. Lift each $\sigma \in S_{i}$ to $\widetilde{\sigma}: \Delta^{i} \rightarrow \widetilde{M}$ and call the union of some choice of lifts $\widetilde{S}_{i}$.

Each $\widetilde{\sigma} \in \widetilde{S_{1}}$ maps its vertices to leaves in $\widetilde{\mathcal{F}}$ which inherit a total order from the partial order on $L$. (Here we actually require the images of distinct vertices of $\Delta^{n}$ to lie on distinct leaves of $\widetilde{\mathcal{F}}$ ). It follows we can replace each $\sigma \in S_{1}$ by a transverse $\sigma^{s}$ with the same endpoints.

Let $\partial \sigma: \partial \Delta^{i} \rightarrow \widetilde{\mathcal{F}}$ be transverse. Then the induced foliation of $\partial \Delta^{i}$ is the standard foliation of the unit sphere $S^{i-1}$ in $\mathbb{R}^{i}$ by its intersection with horizontal planes. The leaves of this foliation are $(i-2)$-spheres away from the top and bottom vertex, with respect to the partial ordering on $L$. Since leaves of $\widetilde{\mathcal{F}}$ are just $\mathbb{R}^{n-1}$ 's, this family of maps of $(i-2)$-spheres extends to a family of maps of $(i-1)$-balls converging at the top and bottom to the image of the top and bottom vertices respectively. This family of maps gives a transverse map $\sigma: \Delta^{i} \rightarrow \widetilde{\mathcal{F}}$ agreeing with $\partial \sigma$ on $\partial \Delta_{i}$.

Thus the straightening procedure can be performed inductively, as required.

Remark 2.2.9. The "nondegeneracy" assumption - that distinct vertices of a simplex get mapped to distinct leaves of $\widetilde{\mathcal{F}}$ under lifts of singular maps $\widetilde{\sigma}$ in the support of $C$ is not really necessary, since our definition of "transverse" cycle includes degenerate affine maps. In any case, any finite chain can be perturbed chain homotopically to a nondegenerate chain without violating the total ordering assumption.

A taut foliation of $M^{3}$ has the properties required by lemma 2.2.8. 
Theorem 2.2.10. Let $\mathcal{F}$ be a foliation of $M^{n}$ whose universal cover is topologically conjugate to the standard foliation of $\mathbb{R}^{n}$ by horizontal $\mathbb{R}^{n-1}$ 's. Then

$$
\|[M]\|_{G}=\|([M], \mathcal{F})\|_{F G}
$$

Proof: $\quad$ Since $\widetilde{\mathcal{F}}$ is the standard foliation of $\mathbb{R}^{n}$ by horizontal planes, the leaf space of $\widetilde{\mathcal{F}}$ is totally ordered. As remarked earlier, we can perturb a chain $C$ by a chain homotopy to a nearby chain $C^{\prime}$ so that for each $\sigma$ in the support of $C, \widetilde{\sigma}$ maps the vertices of $\Delta^{n}$ to distinct leaves of $\widetilde{\mathcal{F}}$. It follows from lemma 2.2.8 that any chain can be straightened to a transverse chain.

In particular, this result holds for $\mathcal{F}$ an $\mathbb{R}$-covered taut foliation of some 3 manifold $M$.

Remark 2.2.11. One easily extends this argument to see that the foliated Gromov norm agrees with the usual Gromov norm for foliations whose universal covering foliations are standard foliations of $\mathbb{R}^{n}$ by horizontal $\mathbb{R}^{n-m}$ 's - that is, for productcovered foliations.

2.3. Measurable chains and equivariant straightening. Theorem 2.2.10 says that finite chains can be straightened with respect to an $\mathbb{R}$-covered foliation. An interesting question is whether the same is true of infinite chains. We make this question more precise.

Definition 2.3.1. Let $\sigma: \Delta^{i} \rightarrow M$ lift to $\widetilde{\sigma}: \Delta^{i} \rightarrow \widetilde{M}$ which can be projected to $\tau: \Delta^{i} \rightarrow L$, the leafspace of $\widetilde{\mathcal{F}}$. $\sigma$ is monotone if the stratification of $\Delta^{i}$ by preimages of points in $L$ is homotopy equivalent to an affine foliation of $\Delta^{i}$. If $\sigma$ is monotone, every $\sigma^{\prime}$ in the support of $\partial \sigma$ is also monotone.

Definition 2.3.2. For each $i$, let $\Sigma_{i}$ denote the space of singular maps $\sigma: \Delta^{i} \rightarrow M$ with the compact-open topology. Let $\Sigma_{i}^{t}$ denote the subspace of transverse singular maps. Let $\Sigma_{i}^{m}$ denote the subspace of monotone singular maps.

In 泪 we prove the following result:

Theorem 2.3.3. Let $\mathcal{F}$ be an $\mathbb{R}$-covered foliation of an atoroidal 3-manifold $M$. Then there are co-ordinates on the universal cover $M=\mathbb{R}^{2} \times \mathbb{R}$ such that leaves of $\widetilde{\mathcal{F}}$ are horizontal planes $\mathbb{R}^{2} \times$ const and $\pi_{1}(M)$ acts by elements of Homeo $\left(\mathbb{R}^{2}\right) \times$ $\operatorname{Homeo}(\mathbb{R})$.

Using this structure, one has at least the following partial result:

Theorem 2.3.4. Let $\mathcal{F}$ be a co-oriented $\mathbb{R}$-covered foliation of a negatively curved closed 3-manifold $M$. Then there are continuous projections from $s_{i}: \Sigma_{i} \rightarrow \Sigma_{i}^{m}$, which are compatible with $\partial$ in the sense that $s_{i-1} \partial=\partial s_{i}$. 
Proof: To avoid cumbersome notation, we define instead straightenings of simplicial maps to $\widetilde{M}$ which are continuous in the compact-open topology, and which are equivariant under the action of $\pi_{1}(M)$.

For each $\sigma: \Delta^{i} \rightarrow \widetilde{M}$ we can define two functions $\rho, \tau$ in terms of the co-ordinates on $\widetilde{M}=\mathbb{R}^{2} \times \mathbb{R}$ by writing

$$
\sigma: t \rightarrow(\rho(t), \tau(t)) \in \mathbb{R}^{2} \times \mathbb{R}
$$

We begin by defining $s_{1}$. Let $\tau(0)=l, \tau(1)=r$ and let $J$ be the set of numbers bounded by $r$ and $l$. There is an obvious retract $\phi: \mathbb{R} \rightarrow J$ which sends everything above $J$ to the maximum of $J$, and everything below $J$ to the minimum of $J$. Set $\tau_{1}(t)=\phi \tau(t)$. Now set

$$
\tau^{\prime}(t)=\inf \left(s: \text { there exists } t_{1} \leq t \leq t_{2} \text { with } \tau_{1}\left(t_{1}\right)=\tau_{1}\left(t_{2}\right)=s\right)
$$

Then $\tau^{\prime}: I \rightarrow \mathbb{R}$ is a monotone map, and we can replace the map $\sigma: t \rightarrow(\rho(t), \tau(t))$ with $s_{1}(\sigma): t \rightarrow\left(\rho(t), \tau^{\prime}(t)\right)$.

Now for $\sigma: \Delta^{2} \rightarrow \widetilde{M}$, we first straighten $\partial \sigma$ using $s_{1}$. The maps $\tau: \partial \Delta^{2} \rightarrow \mathbb{R}$ are already monotone, so for each $p \in \Delta^{2}$, there is a unique $t \in \mathbb{R}$ such that $p$ is in the convex hull of the points in $\partial \Delta$ which are mapped by $\tau$ to $t$. Define $\tau^{\prime}(p)$ to be this value $t$, and set $s_{2}(\sigma): p \rightarrow\left(\rho(p), \tau^{\prime}(p)\right)$.

Finally, for $\sigma: \Delta^{3} \rightarrow \widetilde{M}$, straighten $\partial \sigma$ using $s_{2}$. For each $t$ in the interior of the image of $\left.\tau\right|_{\partial \Delta^{3}}$, the level set $\tau^{-1}(t) \cap \partial \Delta^{3}$ is a cellular subset homotopy equivalent to a circle. It has two frontiers in $\partial \Delta^{3}$, on the side where $\tau$ is greater than $t$ and the side where $\tau$ is less than $t$. Define $D_{t} \subset \Delta^{3}$ to be the minimal surface spanned by the upper frontier of $\tau^{-1}(t)$ (see e.g. [17] for basic facts about minimal surfaces in 3-manifolds). For distinct $s, t$ the disks $D_{t}, D_{s}$ are disjoint, so we can define $\tau^{\prime-1}(t)$ on $\Delta^{3}$ to be the subset of points above $D_{s}$ for all $s<t$ and contained in or below $D_{t}$. Then set $s_{3}(\sigma): p \rightarrow\left(\rho(p), \tau^{\prime}(p)\right)$.

These constructions use only the order structure of $\mathbb{R}$, and are therefore equivariant under the action of $\pi_{1}(M)$.

2.4. The norm is non-trivial on $[M]$. For $M^{n}$ hyperbolic, we know $\|[M]\|_{G}=$ $\operatorname{vol}(M) / v_{n}$ where $v_{n}$ is the volume of the regular ideal $n$-simplex. For a hyperbolic manifold, any cycle can be chain homotoped to a geodesic cycle by replacing each singular map of a simplex $\sigma_{i}: \Delta^{n} \rightarrow M$ with the geodesic simplex $\sigma_{i}^{g}: \Delta^{n} \rightarrow M$ having the same endpoints.

Let $C_{j}$ be a sequence of geodesic chains whose norms converge to the Gromov norm of $M$. Let $\widetilde{C_{j}}$ denote the $\pi_{1}(M)$-equivariant infinite chain obtained by lifting $C_{j}$ to $\widetilde{M}$. Let $X$ be the infinite $(n+1)$-valent tree with basepoint, and let $T$ be 
the abstract complex obtained by gluing together infinitely many ideal $n$-simplices along their faces in the pattern described by $X$. Fix some regular ideal simplex $\Delta \in \mathbb{H}^{n}$. Then choosing an identification of $\Delta$ with some simplex of $T$, there is a natural developing map dev $: T \rightarrow \mathbb{H}^{n}$ taking each simplex of $T$ to a regular ideal simplex. If $n=3$ this has as its image the standard regular tessellation of $\mathbb{H}^{3}$ by ideal simplices. Otherwise, the representation $\operatorname{Aut}(T) \rightarrow \operatorname{Isom}^{+}\left(\mathbb{H}^{n}\right)$ is indiscrete.

Lemma 2.4.1. With notation as above, for any $t, \epsilon>0$ there is a $j$ such that for any $k>j$, there is a collection $S_{k}$ of singular maps in the support of $\widetilde{C_{k}}$ and an element $\alpha_{k} \in \operatorname{Isom}^{+}\left(\mathbb{H}^{n}\right)$ such that $S_{k}$ and $\alpha_{k} \operatorname{dev}(T)$ agree on the ball of radius $t$ about 0 to within $\epsilon$.

Proof: Let $C_{k}=\sum_{i} r_{i} \sigma_{i}$ be a geodesic chain which very nearly realizes the Gromov norm of $M$. Then by definition

$$
\sum_{i}\left|r_{i}\right|<\operatorname{vol}(M) / v_{n}+\delta
$$

for some small $\delta$. On the other hand,

$$
\sum_{i} r_{i} \operatorname{vol}\left(\sigma_{i}\left(\Delta^{n}\right)\right)=\operatorname{vol}(M)
$$

so the weighted average

$$
\frac{\sum_{i} r_{i} \operatorname{vol}\left(\sigma_{i}\left(\Delta^{n}\right)\right)}{\sum_{i}\left|r_{i}\right|}>v_{n}-\delta^{\prime}
$$

for some small $\delta^{\prime}$; that is, "most" of the $\sigma_{i}\left(\Delta^{n}\right)$, as weighted by $r_{i}$, have volume very close to $v_{n}$. This implies that they are geometrically very close to regular ideal simplices, on a big compact set containing most of their mass. (see e.g. [2])

Fix a fundamental domain $D$ in $\widetilde{M}$. For each $\sigma \in \operatorname{supp}\left(C_{k}\right)$, choose a lift $\widetilde{\sigma}$ of $\sigma$ whose center of gravity is in $D$. Since the bundle of frames over $D$ is compact, there is some ideal tetrahedron $\Delta^{\prime}$ in $\mathbb{H}^{n}$ with center of mass in $D$ for which some definite mass of $\widetilde{\sigma}$ is geometrically close to $\Delta^{\prime}$ on a big set. Call $S$ the set of lifts sufficiently close to $\Delta^{\prime}$. It follows that $\partial S$ is geometrically close to $\partial \Delta$. Since $\partial \widetilde{C_{k}}=0$, most of the mass of this boundary must be absorbed by simplices which are geometrically close to being regular and ideal.

It follows that for $\delta$ sufficiently small, we can find lifts $\widetilde{\sigma}$ whose images are close on a big set to the ideal simplices obtained by reflecting $\Delta^{\prime}$ in each of its boundary faces. Let $\alpha_{k}(\Delta)=\Delta^{\prime}$. Continuing inductively, if we propagate outwards in $X$ until we cover a big ball, we can find corresponding simplices in $\operatorname{supp}\left(\widetilde{C_{k}}\right)$ which agree with the simplices in $\alpha_{k} \operatorname{dev}(T)$ to within a suitable tolerance, by taking $\delta$ sufficiently small.

Corollary 2.4.2 (Jungreis). For a hyperbolic $M^{n}$ with $n \geq 3$, a regular ideal simplex $\Delta \subset \mathbb{H}^{n}$ and any sequence $C_{j}$ of geodesic chains whose norms converge to 
$\|M\|_{G}$, for sufficiently large $j$ there is a $\sigma_{j}$ in the support of $C_{j}$ which lifts to a geodesic simplex arbitrarily close to $\Delta$.

Proof: By the previous lemma, there are simplices in the support of $\widetilde{C_{j}}$ which stay close to $\alpha_{k} \operatorname{dev}(T)$ on a big ball about some fixed point. We can identify the set of framed ideal regular simplices in $\mathbb{H}^{n}$ with $\operatorname{Isom}^{+}\left(\mathbb{H}^{n}\right)$. In the limit, the set of ideal regular simplices in the support of $\widetilde{C_{j}}$ is invariant under the action of $\pi_{1}(M)$ on the left and $\operatorname{dev}(A u t(T))$ on the right. If $n>3, \operatorname{dev}(A u t(T))$ is already dense in $\operatorname{Isom}^{+}\left(\mathbb{H}^{n}\right)$. In dimension 3, following Jungreis and Ratner, using both the left and right actions we can find simplices in the support of $C_{j}$ arbitrarily close to $\Delta$ for $j$ sufficiently large. See $[18$ for details.

Definition 2.4.3. Say that a foliation $\mathcal{F}$ of a hyperbolic $n$-manifold is asymptotically separated if for some leaf $\lambda$ of $\widetilde{\mathcal{F}}$, there are a pair of open hemispheres $H^{+}, H^{-} \subset \mathbb{H}^{n}$ in the complement of $\lambda$ which are separated by $\lambda$.

Example 2.4.4. For $\mathcal{F}$ a finite depth foliation which is not a perturbation of a surface bundle over a circle, the compact leaves lift to quasigeodesically embedded planes in $\mathbb{H}^{3}$. Hence every leaf has the separation property, and $\mathcal{F}$ is asymptotically separated.

Theorem 2.4.5. Suppose $M^{n}$ is hyperbolic and $\mathcal{F}$ is asymptotically separated. Then

$$
\|[M]\|_{G}<\|([M], \mathcal{F})\|_{F G}
$$

Proof: By passing to a finite cover if necessary, we can assume that $\mathcal{F}$ is cooriented. If we can show that every chain whose norm is sufficiently close to the Gromov norm contains an edge whose endpoints are not joined by an arc transverse to $\mathcal{F}$, then we will be done.

Let $\lambda$ be a leaf of $\widetilde{\mathcal{F}}$ and $H^{+}, H^{-}$a pair of hemispheres in the complement of $\lambda$ as provided by the definition of asymptotically separated. These determine a pair of disks $D^{+}, D^{-} \subset S_{\infty}^{n-1}$ above and below $\lambda$ respectively. Let $\alpha \in \pi_{1}(M)$ be an element taking the complement of $D^{+}$inside $D^{+}$. Then any infinite line from $D^{-}$ to $\alpha\left(D^{-}\right)$must fail to be transverse to $\widetilde{\mathcal{F}}$ somewhere, since when it crosses $\lambda$ it is going in the positive direction, and when it crosses $\alpha(\lambda)$ it is going in the negative direction, with respect to the co-orientation on $\widetilde{\mathcal{F}}$ which is preserved by $\alpha$.

It is easy to find an ideal regular simplex $\Delta$ which has a pair of endpoints in $D^{-}$and $\alpha\left(D^{-}\right)$respectively. For any chain $C_{j}$ with norm sufficiently close to $\|[M]\|_{G}$, there is a $\sigma$ in the support of $C_{j}$ whose geodesic representative stays very close to $\Delta$ on an arbitrarily large compact piece. Such a $\sigma$ has endpoints on incomparable leaves, and therefore cannot be straightened (keeping its endpoints 
fixed) to a transverse simplex. It follows that no transverse chain can have norm too close to $\|[M]\|_{G}$, and the strict inequality is proved.

2.5. Limit sets of leaves of taut foliations. To investigate the asymptotic separation property, we must investigate the limit sets of leaves of taut foliations.

Lemma 2.5.1. Let $\mathcal{F}$ be taut, and let $\lambda$ be a leaf of $\widetilde{\mathcal{F}}$ on which we have chosen a co-orientation. Denote by $\lambda_{\infty}$ the limit set of $\lambda$. Then each region $D$ in the complement of $\lambda_{\infty}$ is either above or below $\lambda$, in the sense that for any two sequences $\left\{p_{i}\right\} \subset \mathbb{H}^{3}$ and $\left\{q_{i}\right\} \subset \mathbb{H}^{3}$ with $p_{i} \rightarrow p \in D$ and $q_{i} \rightarrow q \in D$, the points $p_{i}, q_{i}$ are eventually on the same side of $\lambda$.

Proof: $\quad$ The points $p$ and $q$ can be joined by an $\operatorname{arc} \alpha$ in $S_{\infty}^{2}$ which avoids $\lambda_{\infty}$. This arc $\alpha$ is the Hausdorff limit in $\mathbb{H}^{3}$ of a sequence of $\operatorname{arcs} \alpha_{i}$ joining $p_{i}$ to $q_{i}$. If each of the $\alpha_{i}$ intersected $\lambda$, this would give rise to a sequence of points in $\lambda$ converging to some point in $\alpha$, contrary to the hypothesis that $\alpha$ avoids $\lambda_{\infty}$. It follows that $p_{i}$ and $q_{i}$ are eventually on the same side of $\lambda$, and therefore the "side" of $D$ is unambiguously defined.

Notice that "above" and "below" as defined in the previous lemma are not the same as $<$ and $>$ in the partial order on $L$. Each leaf in the universal cover of a taut foliation has two sides; a co-orientation on the leaf defines one of the sides to be above and one below, and every other leaf falls into one of these two possibilities. This does not define a partial ordering on leaves.

Definition 2.5.2. Say that a foliation $\mathcal{F}$ has two-sided branching if in the partial order on the leaf space $L$ of $\widetilde{\mathcal{F}}$, there are triples of leaves $\lambda, \lambda_{l}^{+}, \lambda_{r}^{+}$and $\mu, \mu_{l}^{-}, \mu_{r}^{-}$ such that

$$
\begin{gathered}
\lambda<\lambda_{l}^{+}, \lambda<\lambda_{r}^{+} \\
\lambda_{l}^{+} \text {and } \lambda_{r}^{+} \text {are incomparable } \\
\mu_{l}^{-}<\mu, \mu_{r}^{-}<\mu \\
\mu_{l}^{-} \text {and } \mu_{r}^{-} \text {are incomparable }
\end{gathered}
$$

Observe that if $\mathcal{F}$ is taut and has two-sided branching, then we may choose any leaf as $\mu=\lambda$. Moreover, if $\mathcal{F}$ is not co-orientable, or covers some foliation which is not co-orientable, then either $\mathcal{F}$ is $\mathbb{R}$-covered or it has two-sided branching.

Example 2.5.3. Let $\mathcal{F}$ be a foliation of $T^{3}$ with one horizontal torus leaf, and the complementary $T^{2} \times I$ foliated as a Reeb foliation of the annulus $\times S^{1}$. Then a pair of transversals whose initial segments agree and cross the horizontal torus leaf must thereafter be leafwise homotopic; that is, there is no branching in the positive direction from that point on. Thus, we cannot choose $\mu=\lambda$ in this example. 
Example 2.5.4. In [21] G. Meigniez constructs examples of taut foliations which branch on only one side, say the negative side. Furthermore, some of these examples are obtained as perturbations of surface bundles over circles, and therefore have pseudo-Anosov flows transverse to them. In [5] we construct new examples of such foliations, and show that this situation holds in general: taut foliations of atoroidal 3-manifolds with one-sided branching have transverse pseudo-Anosov flows which are regulating: that is, flow lines are properly embedded in the leaf space of the universal cover.

Easy examples of foliations with one-sided branching are obtained by starting with $\mathbb{R}$-covered foliations with (approximately) projectively invariant transverse measures, and then taking branched covers over a curve which lifts to a line in $\widetilde{M}$, one end of which is properly embedded in the leaf space and one end of which is not.

Theorem 2.5.5. Let $\mathcal{F}$ be a taut foliation. If $\mathcal{F}$ has two-sided branching and is not asymptotically separated, then every leaf $\lambda$ of $\widetilde{\mathcal{F}}$ has $m\left(\lambda_{\infty}\right)>0$, where $m$ is some normalized Lebesgue measure on the sphere at infinity $S_{\infty}^{2}$ of $\mathbb{H}^{3}$. In fact, $\lambda_{\infty}$ must have non-empty interior.

Proof: Assume without loss of generality that $\mathcal{F}$ is co-oriented.

Suppose that $m\left(\lambda_{\infty}\right)=0$ for some $\lambda$. Then certainly there is some complementary domain to $\lambda_{\infty}$ in $S_{\infty}^{2}$. If there are domains $D^{ \pm}$both above and below $\lambda$, in the sense of lemma 2.5.1 then there are half-spaces $H^{ \pm}$bounded by circles in $D^{ \pm}$which avoid $\lambda$, and $\mathcal{F}$ is asymptotically separated. Otherwise without loss of generality, all the complementary regions to $\lambda_{\infty}$ are contained above $\lambda$. It follows that the subset of $\widetilde{M}$ below $\lambda$ has limit set exactly equal to $\lambda_{\infty}$.

If $\mathcal{F}$ is $\mathbb{R}$-covered, one knows that $\lambda_{\infty}=S_{\infty}^{2}$ for every $\lambda$, so we may assume $\mathcal{F}$ is not $\mathbb{R}$-covered. (see e.g. [7])

If $\mathcal{F}$ has two-sided branching, then there are a pair of positive transversals to $\widetilde{\mathcal{F}}$ emanating from $\lambda$ and ending on two incomparable translates $\alpha(\lambda), \beta(\lambda)$ both $>\lambda$ in the partial order on $L$. Now, the subset of $\widetilde{M}$ below $\alpha(\lambda)$ has limit set equal to $\alpha\left(\lambda_{\infty}\right)$, which has measure 0 . However, the subset of $\widetilde{M}$ above $\beta(\lambda)$ is itself a subset of the subset of $\widetilde{M}$ below $\alpha(\lambda)$. It follows that we can write $\mathbb{H}^{3}$ as the union of two sets (the sets above and below $\beta(\lambda)$ ), each of which has a limit set of measure 0 , which is absurd. More generally, if $\lambda_{\infty}$ has no interior, we could write $S_{\infty}^{2}$ as the union of two closed sets without interior, which is absurd.

The following theorem is proved in [8]:

Theorem 2.5.6 (Fenley). Let $\mathcal{F}$ be a Reebless foliation in $M^{3}$ closed, hyperbolic. Suppose that $\lambda_{\infty} \neq S_{\infty}^{2}$ for some $\lambda$, and assume that there is branching in the positive and negative directions of $\widetilde{\mathcal{F}}$. Then there is a $k<2$ such that the limit set 
of every leaf has Hausdorff dimension less than $k$. In particular, every such limit set has zero Lebesgue measure.

Corollary 2.5.7. No leaf in the universal cover of a taut foliation of a hyperbolic 3-manifold with two-sided branching is quasi-isometric (as a subset of $\mathbb{H}^{3}$ ) to a totally degenerate surface group.

Proof: The limit set of a totally degenerate surface group is a dendrite: a closed set of measure 0 whose complement is connected (see e.g. [20]). If the limit set of a leaf of a taut foliation has measure 0 , it has at least 2 complementary regions: one above and one below.

Corollary 2.5.8. Let $\mathcal{F}$ have two-sided branching. Then either $\lambda_{\infty}=S_{\infty}^{2}$ for every leaf $\lambda$ of $\widetilde{\mathcal{F}}$ or every leaf is asymptotically separated, and the foliated norm of $[M]$ is strictly greater than the usual norm.

Fenley has conjectured that $\lambda_{\infty}=S_{\infty}^{2}$ iff $\mathcal{F}$ is $\mathbb{R}$-covered.

By contrast, we have the following theorem:

Theorem 2.5.9. Suppose that $\mathcal{F}$ is a taut foliation with one-sided branching. Then there is an equality of norms

$$
\|[M]\|_{G}=\|([M], \mathcal{F})\|_{F G}
$$

Proof: We declare that the branching takes place in the positive direction, with respect to some choice of co-orientation on $\widetilde{\mathcal{F}}$.

Lift the singular maps in the support of a chain $C=\sum_{i} r_{i} \sigma_{i}$ to maps $\widetilde{\sigma}_{i}: \Delta^{3} \rightarrow$ $\widetilde{M}$. It is possible that some vertices of $\Delta^{3}$ are mapped by some $\widetilde{\sigma}_{i}$ to incomparable leaves of $\widetilde{\mathcal{F}}$. However, any pair of points in $\widetilde{M}$ can be made comparable after a finite isotopy in the negative direction. Moreover, if two points are already on comparable leaves, then they are still on comparable leaves after such an isotopy. Since there are only finitely many $\widetilde{\sigma_{i}}$, we can push the images of the vertices under $\sigma_{i}$ in the negative direction to get a new chain, homotopic to $C$, for which each $\widetilde{\sigma}_{i}$ sends the vertices of $\Delta^{3}$ to comparable leaves of $\widetilde{\mathcal{F}}$. By lemma 2.2.8, we can straighten this new chain relative to its vertices to be transverse to $\mathcal{F}$.

Corollary 2.5.10. If $\mathcal{F}$ has one-sided branching, the leaves of $\widetilde{\mathcal{F}}$ are not asymptotically separated.

2.6. Foliations with Reeb components. One might suppose at least that the existence of Reeb components should be detected by the foliated Gromov norm. However, this is not the case, as the following example shows. 
Example 2.6.1. Let $M=S^{2} \times S^{1}$ and $\mathcal{F}$ the standard foliation by two Reeb components. Let $C$ be any chain representing $M$. Let $\pi: M \rightarrow M$ be the unique connected double cover of $M$. Then $\pi^{*} \mathcal{F}=\mathcal{F}$ up to isotopy. However, $\pi_{*} C=2[M]$, so the sequence $2^{-n} \pi_{*}^{n} C$ of chains can be made transverse after isotopy and have norm $\rightarrow 0$. Hence

$$
\|[M]\|_{G}=\|([M], \mathcal{F})\|_{F G}=0
$$

in this case.

Despite this example, it is easy to arrange a sequence of foliations of a given manifold $M$ with more and more Reeb components where the foliated Gromov norm grows without bound, as the following theorem shows.

Theorem 2.6.2. There is a function $k: \mathbb{N} \rightarrow \mathbb{R}$ with $\lim _{n \rightarrow \infty} k(n)=\infty$ such that if $\mathcal{F}$ is any foliation of a 3-manifold $M$ with $n$ generalized Reeb components whose complement is atoroidal, then

$$
\|([M], \mathcal{F})\|_{F G} \geq k(n)
$$

Proof: A generalized Reeb component, also known as a dead end component, is a region of the foliated manifold bounded by torus or Klein bottle compact leaves, such that no path transverse to the foliation which enters the component can leave again.

Let $C=\sum_{i} r_{i} \sigma_{i}$ be a transverse chain representing $[M]$ and let $N \subset M$ be a dead end component. Suppose that $\sigma_{i}$ is a singular simplex whose image intersects $N$. Let $p \in \Delta^{3}$ be such that $\sigma_{i}(p) \in N$ and let $\alpha$ be a path in $\Delta^{3}$ transverse to the foliation induced by $\sigma_{i}^{-1}(\mathcal{F})$ running from the top to the bottom vertices which passes through $p$. Then $\sigma_{i}(\alpha)$ is transverse to $\mathcal{F}$ and intersects $N$; it follows that the image of at least one of the vertices of $\Delta^{3}$ must be contained in $N$.

If we "truncate" $M$ by removing the Reeb components, we get a 3-manifold $M^{\prime}$ with at least $n$ torus or Klein bottle cusps. By the fact above, each truncated simplex can be collapsed to an edge or a face, or else is a normal simplex possibly with some ideal points. The resulting truncated chain $C$ represents $\left[M^{\prime}\right] \in H_{3}\left(M^{\prime}, \partial M^{\prime} ; \mathbb{R}\right)$, so the foliated Gromov norm of $M$ can be estimated by the usual Gromov norm of $M^{\prime}$. Then set $k(n)$ equal to the minimum Gromov norm of a hyperbolic 3-manifold with $n$ cusps.

After the work of Thurston (see e.g. [2]), one knows that $\lim _{n \rightarrow \infty} k(n) \rightarrow \infty$.

\section{Extending The NORM to $H_{*}(M ; \mathbb{R})$}

3.1. Semicontinuity of the norm. It is clear that the definition of the foliated Gromov norm can be extended to a norm on $H_{i}(M ; \mathbb{R})$ for a manifold $M$ foliated by $\mathcal{F}$. As before, for each homology class $\mu$ we can consider transverse singular 
chains representing $\mu$, and take the $L_{1}$ norm of such representatives. Denote the value of this norm on a class $\mu$ by $\|(\mu, \mathcal{F})\|_{F G}$.

Notice that unlike the usual Gromov norm, this norm may be non-trivial even on $H_{1}(M ; \mathbb{R})$, as the following example shows:

Example 3.1.1. Let $\mathcal{F}$ be the foliation of $T^{2} \times I$ obtained by multiplying a Reeb foliation of the cylinder $S^{1} \times I$ by $S^{1}$. Glue the top and bottom of $T^{2} \times I$ together to get a foliation of $T^{3}$ also denoted by $\mathcal{F}$. Let $\alpha \in H_{1}(M ; \mathbb{Z})$ be the generator obtained from the $I$ factor by the gluing. Let $\beta \in H_{1}(M ; \mathbb{R})=r \alpha$. Then $\|(\beta, \mathcal{F})\|_{F G} \geq$ $r / 2$. For, each $\widetilde{\sigma}: \Delta^{1} \rightarrow \mathbb{R}^{3}$ obtained by lifting a map in the support of a chain representing $\beta$ must have length $\leq 2$ in its projection to the vertical factor, since such a chain cannot cross the torus leaf twice.

Theorem 3.1.2. Let $\mathcal{F}_{i}$ be a sequence of taut foliations of $M^{n}$ which converge geometrically (as $(n-1)$-plane fields) to $\mathcal{F}$. Then

$$
\|(A, \mathcal{F})\|_{F G} \geq \lim \sup \left\|\left(A, \mathcal{F}_{i}\right)\right\|_{F G}
$$

for any $A \in H_{*}(M ; \mathbb{R})$.

Proof: $\quad$ Let $C_{j}=\sum_{i} r_{i j} \sigma_{i j}$ be a sequence of cycles transverse to $\mathcal{F}$ representing $A$ whose norms converge to $\|(A, \mathcal{F})\|_{F G}$. Then for any $j$, every $\sigma_{i j}$ is transverse to $\mathcal{F}$ and therefore by compactness there is an $\epsilon_{j}$ such that the 1 -skeleta of the images of $\Delta^{n}$ under the $\sigma_{i j}$ make an angle of at least $\epsilon$ with $\mathcal{F}$ everywhere. It follows that for sufficiently large $k$, the 1 -skeleta of $\operatorname{supp}\left(C_{j}\right)$ is transverse to $\mathcal{F}_{k}$. By lemma 2.2.8 we can straighten $C_{j}$ to be transverse to $\mathcal{F}_{k}$.

This implies that the foliated Gromov norm is lower semi-continuous: the norm can jump up at a limit, but never down. The following example shows, however, that the norm is not actually continuous.

Example 3.1.3. Suppose $M^{3}$ is hyperbolic and fibers over the circle, and has $b_{2} \geq$ 2. Let $\mathcal{F}_{i}$ be a sequence of fiberings contained in some top dimensional face of the Thurston norm converging to some foliation $\mathcal{F}$ which is at a vertex. Then $\left\|\left([M], \mathcal{F}_{i}\right)\right\|_{F G}=\|[M]\|_{G}$ by theorem 2.2.10. On the other hand, $\mathcal{F}$ contains a quasigeodesically embedded compact leaf, so $\|([M], \mathcal{F})\|_{F G}>\|[M]\|_{G}$ by theorem 2.4.5.

An interesting phenomenon in the theory of foliations occurs when a sequence of isotopies of a fixed foliation $\mathcal{F}$ converges geometrically to a topologically distinct foliation $\mathcal{G}$. We give a simple example of this phenomenon.

Example 3.1.4. Let $S$ be the cylinder $I \times S^{1}$ foliated by horizontal circles point $\times S^{1}$. For an end-preserving homeomorphism $f: I \rightarrow I$ we can produce a foliation $\mathcal{F}_{f}$ of $T^{2} \times I$ which is the suspension of the foliation of $S$ by the map $f \times$ id $: S \rightarrow S$. Any 
two topologically conjugate maps $f, g: I \rightarrow I$ give isotopic foliations. Now, it is well-known that any two strictly increasing homeomorphisms of the open interval to itself are topologically conjugate. One can easily find a sequence $f_{i}$ of these which converge (as maps $I \rightarrow I$ ) to the identity. The foliations $\mathcal{F}_{f_{i}}$ are all isotopic, but distinct from $\mathcal{F}_{\text {id }}$.

In any case, upper-semicontinuity of the norm implies the following

Corollary 3.1.5. Let $\mathcal{F}, \mathcal{G}$ be taut foliations of $M$ and suppose

$$
\|(A, \mathcal{F})\|_{F G}>\|(A, \mathcal{G})\|_{F G}
$$

for some $A$. Then no sequence of isotopies of $\mathcal{F}$ can converge geometrically to $\mathcal{G}$.

Dual to the $L_{1}$ norm on $C_{*}(M ; \mathbb{R})$ defined by a foliation, there is an $L_{\infty}$ norm on $C^{*}(M ; \mathbb{R})$ defined as the supremum of the value of the cochain on transverse singular maps. There is an associated foliated bounded cohomology, denoted by $H_{\mathcal{F}}^{*}(M ; \mathbb{R})$. This may contain nontrivial elements even in dimension 1 , in contrast with the usual bounded cohomology.

3.2. Length of a free homotopy class. There is a homotopy-theoretic refinement of the norm on $H_{1}$. Say that a free homotopy class $[\alpha]$ of loops is transverse to $\mathcal{F}$ if $\alpha$ is freely homotopic to a transverse circle. More generally, define the length of $[\alpha]$, denoted $\ell([\alpha])$, to be the minimum number of subdivisions of $S^{1}$ needed to make a representative transverse on each subdivision. Say that this length is 0 if no subdivision is necessary: that is, if some loop representing $\alpha$ is either transverse as a circle to $\mathcal{F}$ or can be homotoped into a leaf of $\mathcal{F}$.

Notice that for a co-oriented foliation, $\ell$ takes on only even values.

Lemma 3.2.1. The length of a free homotopy class of loops $[\alpha]$ is upper semicontinuous in the geometric topology.

Proof: The only non-obvious point to check is that if $[\alpha]$ has a representative which is contained in a leaf of $\mathcal{F}$, then $\ell([\alpha])=0$ for any $\mathcal{G}_{i}$ sufficiently close to $\mathcal{F}$ in the geometric topology. Lift $\alpha$ to an arc $\widetilde{\alpha}$ in $\widetilde{M}$ contained in a leaf of $\widetilde{\mathcal{F}}$. Then there is a big ball $B$ containing $\widetilde{\alpha}$ which is foliated in a standard way by $\widetilde{\mathcal{F}}$. Therefore, for any $\mathcal{G}_{i}$ sufficiently close to $\mathcal{F}$ in the geometric topology, $\widetilde{\mathcal{G}}_{i}$ foliates some slightly smaller ball, also containing $\alpha$, in a standard way. The arc $\alpha$ may be made transverse or tangential to $\widetilde{\mathcal{G}_{i}}$ in this ball, implying that $\ell([\alpha])=0$ for $\mathcal{G}_{i}$.

Lemma 3.2.2. For any co-oriented taut foliation $\mathcal{F}$ which has two-sided branching, there is an $[\alpha] \in \pi_{1}(M)$ with $\ell([\alpha]) \geq 2$.

Proof: Let $\tau_{1}, \tau_{2}$ be two positive transversals to $\widetilde{\mathcal{F}}$ emanating from the same point whose upper endpoints are on incomparable leaves. Let $\sigma_{1}, \sigma_{2}$ be two negative 
transversals to $\widetilde{\mathcal{F}}$ emanating from the same point whose lower endpoints are on incomparable leaves.

Map $\tau_{1} \cup \tau_{2}$ and $\sigma_{1} \cup \sigma_{2}$ downstairs to $M$. Since $\mathcal{F}$ is taut, one can extend the image of $\tau_{1}$ in the positive direction by an arc $\rho_{1}$ until it joins up with $\sigma_{1}$, and do the same with $\tau_{2}$. The union makes up a loop $\alpha$, consisting of two transverse arcs $\alpha_{1}=\tau_{1} \cup \rho_{1} \cup \sigma_{1}$ and $\alpha_{2}=\tau_{2} \cup \rho_{2} \cup \sigma_{2}$. We orient $\alpha$ so that $\alpha_{1}$ is positive and $\alpha_{2}$ is negative. Let $\widetilde{\alpha}$ be a lift to $\widetilde{M}$, and consider its projection to the leaf space $L$ of $\widetilde{\mathcal{F}}$ : this consists of an alternating sequence of positive and negative arcs, which pass over a branch of $L$ at each stage. If $t_{i}$ and $b_{i}$ denote the alternating sequence of top and bottom leaves of the projection, then for all $i \in \mathbb{Z}$,

$$
t_{i}>b_{i}, t_{i}>b_{i-1}
$$

$b_{i}$ and $b_{i+1}$ are incomparable

$t_{i}$ and $t_{i+1}$ are incomparable

We can find a sequence of points $m_{i}$ with $b_{i}<m_{i}<t_{i}$ and each $m_{i}, b_{i-1}$ and $m_{i}, t_{i+1}$ pairwise incomparable. It is clear that for any $\alpha^{\prime}$ homotopic to $\alpha$, the projection of the corresponding lift $\widetilde{\alpha^{\prime}}$ to $L$ must intersect each $m_{i}$. In particular, such a lift intersects incomparable leaves of $\widetilde{\mathcal{F}}$, so $\alpha^{\prime}$ cannot be transverse. See figure 3 .

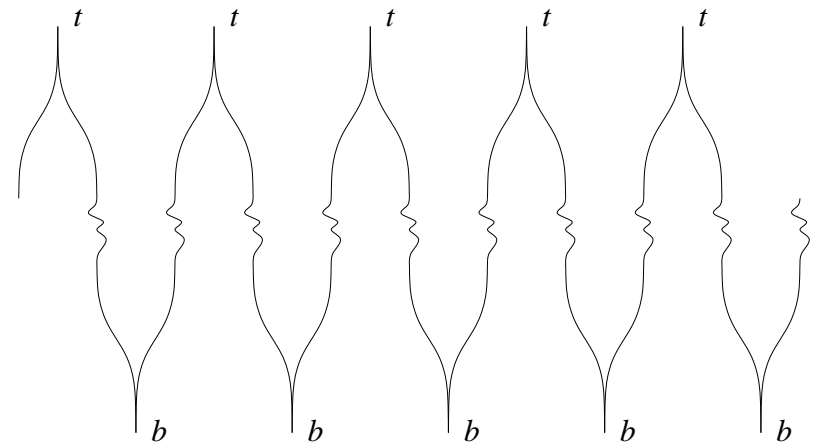

Figure 3. The sequence of lifts of $\alpha_{1}$ and $\alpha_{2}$, projected to $L$ alternately branches in the positive and negative direction; the same is true of any homotopic lift.

The following corollary answers a question posed by W. Thurston:

Corollary 3.2.3. Let $\mathcal{F}$ with branching on at most one side and $\mathcal{G}$ with two-sided branching, be taut foliations of $M$. Then there is no sequence of isotopies $\mathcal{G}_{i}$ of $\mathcal{G}$ which converges geometrically to $\mathcal{F}$.

Proof: Lift to a finite cover where the foliations are co-oriented. Then observe that for a foliation with branching on at most one side, the length of any free 
homotopy class is 0 . For, suppose $\mathcal{F}$ does not branch in the positive direction, and let $\alpha$ be any loop in $M$, and suppose $\alpha$ is in general position with respect to $\mathcal{F}$ so that it has a finite number of isolated minima and maxima. Let $p$ be such a minimum, lying between maxima $q, r$. Lift the segment $\tau$ between $q, r$ to $\widetilde{\tau}$ in $\widetilde{M}$. Then $\widetilde{q}, \widetilde{r}$ are comparable, since they are both $>\widetilde{p}$ and by hypothesis there is no branching in the positive direction, so without loss of generality we can assume $\widetilde{q} \leq \widetilde{r}$. So we can push the local minima corresponding to $p$ in the positive direction until it cancels the local maxima corresponding to $q$ without introducing any new critical points; in particular, the number of critical points can be reduced. Continuing inductively, they can all be eliminated.

\subsection{Virtually fine triangulations.}

Definition 3.3.1. Let $M$ be a 3-manifold. A triangulation $\tau$ of $M$ is fine if for every taut foliation $\mathcal{F}$ of $M \tau$ can be isotoped to be transverse to $\mathcal{F}$. A triangulation $\tau$ is virtually fine if for every taut foliation $\mathcal{F}$ of $M$ there is a finite cover $\widehat{M}$ of $M$ such that the pulled-back triangulation $\widehat{\tau}$ can be isotoped to be transverse to the pulled-back foliation $\widehat{\mathcal{F}}$.

One of the main theorems of [11] states that for any $M$ there is a fine triangulation $\tau$. This leads naturally to the question of what conditions are necessary and sufficient on a triangulation to be fine. An obvious condition is that the triangulation admit a transverse foliation locally. For a geodesic triangulation of a hyperbolic manifold, this is obvious, since in the projective model of hyperbolic space, a hyperbolically geodesic triangulation looks like a Euclidean triangulation of the ball, and a foliation by horizontal planes will be transverse. It has been an open question whether every geodesic triangulation of a hyperbolic 3-manifold $M$ is virtually fine.

It turns out this guess is incorrect: there are geodesic triangulations whose simplices have diameters arbitrarily small compared to the injectivity radius of the ambient manifold, which cannot be made transverse to certain taut foliations.

Theorem 3.3.2. Let $M$ be a hyperbolic 3-manifold, and $\mathcal{F}$ any taut foliation with 2 -sided branching. Then there is a geodesic triangulation $\tau$ of $M$ which cannot be made transverse to $\mathcal{F}$. Furthermore, $\tau$ cannot be made transverse to $\mathcal{F}$ in any finite cover (i.e. $\tau$ is not virtually fine).

Proof: $\quad$ Let $\gamma$ be a closed loop with $\ell(d)=l \geq 2$. Then we can choose a geodesic representative of $\gamma$ and make it an edge of a geodesic triangulation. Such a triangulation can obviously not be made transverse to $\mathcal{F}$. Now, for any finite cover $\widehat{M}$ of $M$, we can lift $\gamma$ to some $\widehat{\gamma}$ which covers $\gamma$ with degree $d$, and has $d$ segments in the lifted triangulation. With respect to $\widehat{\mathcal{F}}$, the new length of $\widehat{\gamma}$ is $l d$, so the lifted triangulation cannot be made transverse to the lifted foliation. 


\section{LAMINATIONS AND ORDER TREES}

4.1. Genuine laminations. Laminations of 3 -manifolds are defined in [13].

Definition 4.1.1. A lamination in a 3 -manifold is a foliation of a closed subset of $M$ by 2 dimensional leaves. The complement of this closed subset falls into connected components, called complementary regions. A lamination is essential if it contains no spherical leaf or torus leaf bounding a solid torus, and furthermore if $C$ is the closure (with respect to the path metric in $M$ ) of a complementary region, then $C$ is irreducible and $\partial C$ is both incompressible and end incompressible in $C$. Here an end compressing disk is a properly embedded $\left(D^{2}-\left(\operatorname{closed}\right.\right.$ arc in $\left.\left.\partial D^{2}\right)\right)$ in $C$ which is not properly isotopic relative to the $\partial$ in $C$ to an embedding into a leaf. Finally, an essential lamination is genuine if it has some complementary region which is not an $I$-bundle.

An essential lamination simultaneously generalizes both Reebless foliations and incompressible surfaces. It is not true that an essential lamination lifts in a finite cover to a co-orientable lamination. Consequently the leaf space of an essential lamination in the universal cover does not carry a natural partial order. The leaf space of a foliation is like a train-track: there is a natural combing near any branch point. The leaf space of a lamination is more like a tree: there is no natural way to say whether branches approach a branch point from the same or from opposite directions.

Nevertheless, we can still talk about transversality of a simplicial map in a laminated context.

Definition 4.1.2. Let $\Lambda$ be an essential lamination of $M$. A map $\sigma: \Delta^{1} \rightarrow M$ is transverse if there is no back-tracking; i.e. there is no subinterval of $\Delta^{1}$ whose image can be homotoped relative to its endpoints into a leaf of $\Lambda$. A map $\sigma: \Delta^{i} \rightarrow M$ with $i \geq 2$ is transverse if the induced lamination $\sigma^{-1}(\Lambda)$ of $\Delta^{i}$ is non-singular and can be completed to an affine foliation of $\Delta^{i}$

Denote by $\|(A, \Lambda)\|_{L G}$ the norm of a homology class $A$ with respect to $\Lambda$.

For $\sigma: \Delta^{3} \rightarrow M$ a transverse map and $\Lambda$ nowhere dense, we can perturb $\sigma$ to be nondegenerate; that is, so that $\sigma^{-1}(\Lambda)$ is a collection of normal triangles and quadrilaterals compatible with a total ordering of the vertices of $\Delta^{3}$. This can be done by wiggling $\sigma$ slightly so that no vertex is taken into a leaf of $\Lambda$.

Let $\mathcal{T}$ be a triangulation of $M$, and let $n(\mathcal{T})$ denote the number of tetrahedra in $\mathcal{T}$. Then any minimal genuine lamination $\Lambda$ (i.e. one with every leaf dense in $\Lambda$ ) can be put in normal form with respect to $\mathcal{T}$, by a theorem of M. Brittenham 3. For instance, an incompressible surface is an example of a minimal lamination. This establishes the following estimate 
Theorem 4.1.3. Let $\Lambda$ be a minimal genuine lamination of $M$. Then

$$
\|([M], \Lambda)\|_{L G} \leq \min _{\mathcal{T}} 4 n(\mathcal{T})
$$

Proof: Let $\mathcal{T}$ be a triangulation of $M$. Then we can isotope $\Lambda$ to be in normal form with respect to $\mathcal{T}$. Now we can subdivide $\mathcal{T}$, replacing each tetrahedron by 4 tetrahedra, each of which only contains normal disks compatible with a total ordering on its vertices.

On the other hand, corollary 2.4 .2 implies

Theorem 4.1.4. For $S$ an incompressible surface in a hyperbolic 3-manifold $M$, either $S$ is a fiber of a fibration of $M$ over $S^{1}$ or

$$
\|([M], S)\|_{L G}>\|[M]\|_{G}
$$

Proof: Either $S$ is a fiber of a fibration over $S^{1}$, or $S$ is quasigeodesic. In the second case, we can find three lifts $\widetilde{S}_{1}, \widetilde{S}_{2}, \widetilde{S}_{3}$ of $S$ to $\widetilde{M}=\mathbb{H}^{3}$ which bound disjoint half-spaces. There is an ideal triangle with one vertex in each of these half-spaces, and there is a regular ideal tetrahedron, one of whose faces is this triangle. It follows that any chain representing $[M]$ whose norm is sufficiently close to $\|[M]\|_{G}$ cannot be transverse to $S$.

4.2. Order trees. The following definition is found in 13$]$.

Definition 4.2.1. An order tree is a set $T$ together with a collection $\mathcal{S}$ of linearly ordered subsets called segments, each with distinct least and greatest elements called the initial and final ends. If $\sigma$ is a segment, $-\sigma$ denotes the same subset with the reverse order, and is called the inverse of $\sigma$. The following conditions should be satisfied:

- $\sigma \in \mathcal{S} \Longrightarrow-\sigma \in \mathcal{S}$

- Any closed subinterval of a segment is a segment (if it has more than one element).

- Any two elements of $T$ can be joined by a finite sequence of segments $\sigma_{i}$ with the final end of $\sigma_{i}$ equal to the initial end of $\sigma_{i+1}$.

- Given a cyclic word $\sigma_{0} \sigma_{1} \ldots \sigma_{k-1}$ (subscripts $\bmod k$ ) with the final end of $\sigma_{i}$ equal to the initial end of $\sigma_{i+1}$, there is a subdivision of the $\sigma_{i}$ yielding a cyclic word $\rho_{0} \rho_{1} \ldots \rho_{n-1}$ which becomes the trivial word when adjacent inverse segments are canceled.

- If $\sigma_{1}$ and $\sigma_{2}$ are segments whose intersection is a single element which is the final element of $\sigma_{1}$ and the initial element of $\sigma_{2}$ then $\sigma_{1} \cup \sigma_{2}$ is a segment containing $\sigma_{1}$ and $\sigma_{2}$. 
An order tree is topologized by the order topology on segments. We assume in the sequel that our order trees are $\mathbb{R}$-order trees - that is, 2nd countable order trees whose segments are order isomorphic to compact intervals of $\mathbb{R}$.

Let $\Gamma=\pi_{1}(M)$ act by automorphisms on an order tree $T$ and suppose that we have a $\Gamma$-equivariant surjective map

$$
\phi: \widetilde{M} \rightarrow T
$$

A singular map $\sigma: \Delta^{i} \rightarrow M$ is transverse if for any lift $\widetilde{\sigma}: \Delta^{i} \rightarrow \widetilde{M}$ the composition $\phi \widetilde{\sigma}: \Delta^{i} \rightarrow T$ maps $\Delta^{i}$ to a totally ordered segment of $T$.

Say that a sequence of such maps $\phi_{i}: \widetilde{M} \rightarrow T_{i}$ converges to $\phi: \widetilde{M} \rightarrow T$ if every map $\sigma: \Delta \rightarrow M$ transverse with respect to $\phi$ is eventually transverse with respect to $\phi_{i}$, for sufficiently large $i$.

For a representation $\rho: \Gamma \rightarrow \operatorname{Aut}(T)$, an equivariant map $\phi: \widetilde{M} \rightarrow T$ and a homology class $\mu \in H_{i}(M ; \mathbb{R})$ we can define a norm $\|(\mu, \phi)\|_{F G}$ as before as the $L_{1}$ norm on the singular chains representing $\mu$ which are transverse with respect to $\phi$. Observe that this norm does not really depend on the map $\phi$, since it is determined up to equivariant homotopy by $\rho$, and thus this is really a norm on $H_{i}(\Gamma ; \mathbb{R})$ depending only on $\rho$.

Theorem 4.2.2. Let $\Gamma=\pi_{1}(M)$ and let $\phi_{i}: \widetilde{M} \rightarrow T_{i}$ be a sequence of equivariant maps for actions $\rho_{i}: \Gamma \rightarrow$ Aut $(T)$. Suppose this sequence converges to $\phi: \widetilde{M} \rightarrow T$ equivariant for some action $\rho: \Gamma \rightarrow A u t(T)$.

Then given $\mu \in H_{i}(M ; \mathbb{R})$ we have the inequality

$$
\|(\mu, \phi)\|_{F G} \geq \limsup \left\|\left(\mu, \phi_{j}\right)\right\|_{F G}
$$

Proof: $\quad$ Any geometric chain in $M$ representing $\mu$ which is transverse for $\phi$ will be transverse for $\phi_{i}$ for sufficiently large $i$.

Examples of group actions on order trees arise in the study of essential laminations, where the lamination in the universal cover is dual to an order tree which can be taken to be an $\mathbb{R}$-order tree by replacing isolated leaves by foliated $I$-bundles over those leaves. We have already seen from the example of foliations that this norm is not trivial and can vary for different representations of a fixed group. 


\section{REFERENCES}

[1] I. Agol, Lower bounds on volumes of hyperbolic Haken 3-manifolds, math.GT/9906182

[2] R. Benedetti and C. Petronio, Lectures on hyperbolic geometry, Springer-Verlag Universitext (1992)

[3] M. Brittenham, Essential laminations and Haken normal form, Pacific J. Math. 168 no. 2 (1995), pp. 217-234

[4] D. Calegari, The geometry of $\mathbb{R}$-covered foliations I, math.GT/9903173

[5] D. Calegari, Foliations with one-sided branching, preprint.

[6] M. Culler and P. Shalen, Varieties of group representations and splittings of 3-manifolds, Ann. of Math. (2) 117 (1983), pp. 109-146

[7] S. Fenley, Quasi-isometric foliations, Topology 31 no. 3 (1992), pp. 667-676

[8] S. Fenley, Limit sets of foliations in hyperbolic 3-manifolds, Topology 374 (1998), pp. 875894

[9] D. Gabai, Foliations and the topology of 3-manifolds, J. Diff. Geom. 18 no.3 (1983), pp. 445-503

[10] D. Gabai, Problems in foliations and laminations, in Geometric Topology part 2, proceedings of 1993 Georgia International Topology Conference, ed. W. Kazez (1997), pp. 1-33

[11] D. Gabai, Essential laminations in Kneser normal form, preprint; to appear in J. Diff. Geom.

[12] D. Gabai and W. Kazez, Order trees and laminations of the plane, Math. Res. Let. 4 no. 4 (1997), pp. 603-616

[13] D. Gabai and U. Oertel, Essential laminations in 3-manifolds, Ann. of Math. (2) 130 (1989), pp. $41-73$

[14] M. Gromov, Volume and bounded cohomology, Inst. Hautes Études Sci. Publ. Math. no. 56 (1982), pp. 5-99

[15] M. Gromov, Foliated Plateau problem I, Geometric and functional analysis 1 no.1 (1991), pp. $14-80$

[16] M. Gromov, Metric structures for Riemannian and Non-Riemannian spaces, Birkhauser (1999)

[17] J. Hass, Minimal surfaces in foliated manifolds, Comment. Math. Helv. 61 (1986), pp. 1-32

[18] D. Jungreis, Chains that realize the Gromov invariant of hyperbolic manifolds, Ergodic Theory Dynam. Systems 17 no. 3 (1997), pp. 643-648

[19] R. Kirby, Problems in low-dimensional topology, in Geometric Topology part 2, proceedings of 1993 Georgia International Topology Conference, ed. W. Kazez (1987), pp. 35-473

[20] C. McMullen, Renormalization and 3-manifolds which fiber over the circle, Ann. Math. Studies 142 (1996)

[21] G. Meigniez, Bouts d'un groupe opérant sur la droite: 2. Applications à la topologie des feuilletages, Tôhoku Math. J. 43 (1991), pp. 473-500

[22] F. Paulin, Topologie de Gromov équivariante, structures hyperboliques et arbres réels, Invent. Math. 94 no. 1 (1988), pp. 53-80

[23] J. Stallings, A topological proof of Grushko's theorem on free products, Math. Z. 90 (1965), pp. $1-8$

[24] W. Thurston, The geometry and topology of 3-manifolds (a.k.a "Thurston's notes"), preprint.

Department of Mathematics, Harvard, Cambridge, MA 02138

E-mail address: dannyc@math.harvard.edu 